\title{
Elastic properties of DNA revealed
}

A neat technique originally devised for straightening molecules of DNA tums out to be an effective way of measuring the elastic properties of the molecule.

WITH the passage of time, virtually every aspect of the properties of the archetypal DNA molecule will have been investigated. For such an important structure, nothing less can be expected. But there may be particular interest in a way of measuring the mechanical strength of duplex DNA molecules that has now come to light. At this stage, the numerical result may be less important than the neat method by which it has been obtained, and which may have more general usefulness in the investigation of single molecules.

How can such a measurement be accomplished? The first need is evidently a way of anchoring a single molecule, a question amply explored in the past few years because of the interest in the uses of tethered oligonucleotide fragments of defined and different structure as means of capturing complementary polynucleotides from solution. It remains to be seen whether such a technique will offer advantages in the rapid sequencing of DNA, but it cannot be long before "DNA on a chip" is used in diagnostic kits of various kinds. (The purchase by Glaxo of the company called Affymax refers.)

But even when a molecule of DNA has been anchored to a solid surface, it is far from obvious how to apply to it a steadily increasing force that is not so great that it pulls the molecule apart immediately. The old school-laboratory experiment for the measurement of Young's Modulus, in which weights are added in succession to the bottom of wire hung from, say, a ceiling, is the model. A group in Paris has found an ingenious solution for molecules of DNA: anchor a molecule at both ends to a solid surface, immerse the whole in an aqueous solution and then withdraw the liquid slowly, so that the middle section of the DNA molecule is pulled away from its anchorages by the retreating meniscus of the liquid.

The advantage of this arrangement is that the force exerted on the molecule is self-limiting. As the meniscus retreats, it feeds out extra DNA as the reel on a fishing-rod releases extra line. At least in the early stages of the process, the molecule is unlikely to snap physically. The designers of this arrangement are D. Bensimon, A.J. Simon and V. Croquette from the Ecole Normale Supérieure and A. Bensimon from the Institut Pasteur (Phys. Rev. Letts 74, 4754-4757; 1995). A similar procedure, called "molecular combing", is applied to DNA molecules anchored at one end only to pull them straight and align them in one direction on a solid surface.

In the established tradition of most studies of the physical properties of DNA, Bensimon and colleagues have worked with the DNA of bacteriophage $\lambda$. There are several ways of anchoring DNA molecules to surfaces, which must be coated in advance with suitable anchorage material. The essential requirement is that DNA molecules should be able to bind only at their ends. To make them visible, the molecules are stained with a fluorescent dye.

Even the control experiments the Paris group describes are telling. In combing DNA molecules anchored only at one end, it turns out that the average length of a $\lambda$-molecule amounts to $24 \mu \mathrm{m}$ when the surface carries a hydrophobic coating (such as a silane), but is about two-thirds as much when the surface is hydrophylic. The inference is that the retreating meniscus can more easily stretch an anchored DNA molecule when the molecule has no particular affinity for the underlying surface than when the affinity for the surface is strong, as it must be when the surface is hydrophylic. The actual numbers show that $\lambda$-DNA can be extended by at least 50 per cent without snapping.

The stretching of doubly anchored $\lambda$-DNA by a retreating meniscus is more revealing. What then seems to happen is that, after the meniscus has retreated beyond both anchorage points, the length of the molecular tether to each anchorage point is extended in a direction perpendicular to the retreating interface. Only when the length of the DNA within the retreating liquid is equal to the distance between the two legs do unexpected things happen.

Then, it is not difficult to imagine that it will no longer be possible for the two legs of the molecule to be extended in a direction perpendicular to the meniscus, and that the tension along the length of the DNA molecule will at this stage be steadily increased. After the meniscus has left the whole molecule high and dry, the result is a shape in which two parallel legs are joined by a roughly semi-circular shape, making an overall shape not very different from a vertical cross-section through an old-fashioned test-tube.

According to Bensimon et al., the remarkable feature of these shapes is that (for the same surface coverings) they are identical except for a scale factor determined by the distance between the two parallel legs. Another, vividly apparent from the photographs accompanying their article, is that the DNA molecule may sometimes snap and that, if it does, the break will appear at the curved end of the loop and will be made apparent by two dangling lines of fluorescence conveniently aligned in the direction of the retreating meniscus.

From that phenomenon, it is possible to estimate the degree to which DNA can be stretched without breaking. The argument goes like this: in a curve-ended loop of DNA with two dangling pieces of DNA, the width of the gap presumably represents the length of the fully-stretched DNA, while the two dangling pieces are the unstretched length of the same DNA segment. By using molecular combing as a way of calibrating these measurements, Bensimon et al. conclude that a DNA molecule can be stretched to roughly twice its native length before snapping.

By any standards, that estimate (the average of 25 measurements) is surprisingly high. How the normal duplex configuration could allow such great extension are not easily pinned down. Presumably an untwisting of the helix over relatively short lengths of an entire molecular would be energetically more favourable than a separation of the two antiparallel strands, but the point would bear investigation. Meanwhile, it also emerges that the shape left by a doubly anchored molecule from which the meniscus has retreated can be calculated explicitly, leading to formal expressions for the shape of the fluorescent molecules left behind by a retreating meniscus that differ only in the value of a single parameter. For a particular value of this constant, the blunt end of an unbroken doubly-anchored molecule is accurately a semicircle. In principle, it should be possible to turn this argument around, and to calculate from the shape of a doubly anchored molecule (whether or not it snaps) the controlling elements of any particular measurement, of which the chief seems to be the affinity of stranded DNA for the underlying surface.

The numbers have some interest. Young's modulus, for what it is worth, turns out to be $1.1 \times 10^{8} \mathrm{~N} \mathrm{~m}^{-2}$. But that must be a composite figure; with such a structure, Young's modulus should vary with the strain. The force needed to break a DNA molecule is estimated at 480 pico-Newtons, but with an error of 16 per cent. (From that, it should readily be apparent that a $1 \mathrm{~mm}$ thread of DNA would support a weight of 120 kilograms.)

John Maddox 